\title{
A Critical Appraisal of the Box Counting Method to Assess the Fractal Dimension of Tree Crowns
}

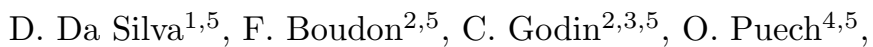 \\ C. Smith $^{4,5}$, and H. Sinoquet ${ }^{6}$ \\ ${ }^{1}$ Université de Montpellier II ${ }^{2}$ CIRAD ${ }^{3}$ INRIA ${ }^{4}$ INRA \\ ${ }^{5}$ Virtual Plants Team, UMR AMAP TA/40E 34398 Montpellier Cedex 5, France \\ ${ }^{6}$ INRA-UBP, UMR PIAF, \\ Domaine de Crouelle, 234 avenue du Brézet 63100 Clermont-Ferrand, France
}

\begin{abstract}
In this paper, we study the application of the box counting method (BCM) to estimate the fractal dimension of 3D plant foliage. We use artificial crowns with known theoretical fractal dimension to characterize the accuracy of the BCM and we extend the approach to $3 \mathrm{D}$ digitized plants. In particular, errors are experimentally characterized for the estimated values of the fractal dimension. Results show that, with careful protocols, the estimated values are quite accurate. Several limits of the BCM are also analyzed in this context. This analysis is used to introduce a new estimator, derived from the BCM estimator, whose behavior is characterized.
\end{abstract}

\section{Introduction}

Plant geometry is a key factor for modeling eco-physiological interaction of plant and the environment. These interactions may concern either the abiotic (resource capture, heat dissipation) or the biotic (disease propagation, insect movement) environment. Depending on applications, plant geometry has been abstracted in various ways [1] : simple volumic shapes (like ellipsoids, cones, or big leaves used in turbid medium approaches) or detailed models to render realistic trees. Global descriptions are simple and contain few parameters; however, they do not capture the irregular nature of plant shapes which severely limits the generalization capacity of the model. On the other hand, detailed descriptions tentatively address this problem but require over-parameterization of geometry, leading to non-parsimonious models. Characterizing the irregularity of plant shapes with a few parameters is thus a challenging problem.

ractal geometry was introduced as a new conceptual framework to analyze and model the irregular nature of irregular shapes [2]. This framework has been applied in different occasions to the modeling of plant structure. Generative approaches use fractal concepts to illustrate how intricate vegetal-like structures can be generated using parsimonious models 345. Such models were used to generate artificial plants in modeling applications 6 67. Fractal geometry was 
also used to analyze the irregularity of plants by determining their supposed fractal dimension. This parameter is of major importance in the study of irregularity: it characterizes the way plants physically occupy space. Most of these studies were carried out using the classical box counting method (BCM) [2] on woody structures, and especially on root systems 86910. This method consists of immersing the studied object in a grid with uniform cell size and studying the variation of the number of grid cells intercepted by the plant as the size of the cells decreases.

For practical reasons, in most works, fractal dimension is estimated from 2D photographs 1112. Unfortunately, such a technique always under-estimates the actual fractal dimension [13], and so is not accurate. Recently BCM was used on 3D digitized root systems [10; however, the accuracy of the estimated values could not be evaluated. In this paper, we study the application of the BCM to both artificial and real 3D plant foliage. We use artificial crowns with known theoretical fractal dimensions to characterize the accuracy of BCM and we extend the approach to 3D digitized plants. The limits of BCM is then analyzed and discussed in this context.

\section{Plant Databases}

Nine 3D plants were included in the study. Four real trees were digitized in the field and five additional plants were generated from theoretical models. The geometric scenes representing the plant crowns were designed using the PlantGL library 14.

Digitized Plants. Four four-year old Prunus Persica (peach) trees were digitized [15, but due to the high number of leaves $(\sim 14,000)$, digitizing at leaf scale was impossible. A magnetic digitizing device was therefore used to record the spatial co-ordinates of the bottom and top of each leafy shoot. In addition, thirty shoots were digitized at leaf scale in order to derive the leaf angle distribution, and allometric relationships between number of leaves, shoot leaf area and shoot length. Leaves of each shoot were then generated from those data and additional assumptions for the internode length and the distribution of leaf size within a shoot.

Theoretical Plants. Three fractal plants were generated from 3D iterated function systems (IFS) [4. The generation process is illustrated in Fig. 2, and the finals artificial canopies are represented in Fig. 3. If the IFS satisfies the open set condition [16], the theoretical fractal dimension of the IFS attractor is the autosimilarity dimension,

$$
D_{a}=\frac{\log n}{\log c}
$$

A classical 3D cantor dust 2] was also generated using an IFS $(n=8, c=3)$. Each IFS was developed over 5 iterations. In addition to these self-similar plants 

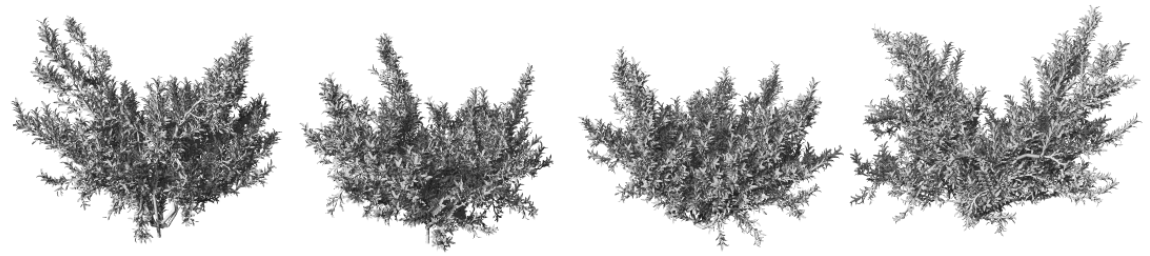

Fig. 1. Four four-year old peach trees (cv. August Red) were digitized in May 2001 in CTIFL Center, Nîmes, South of France, at current-year shoot scale, one month after bud break

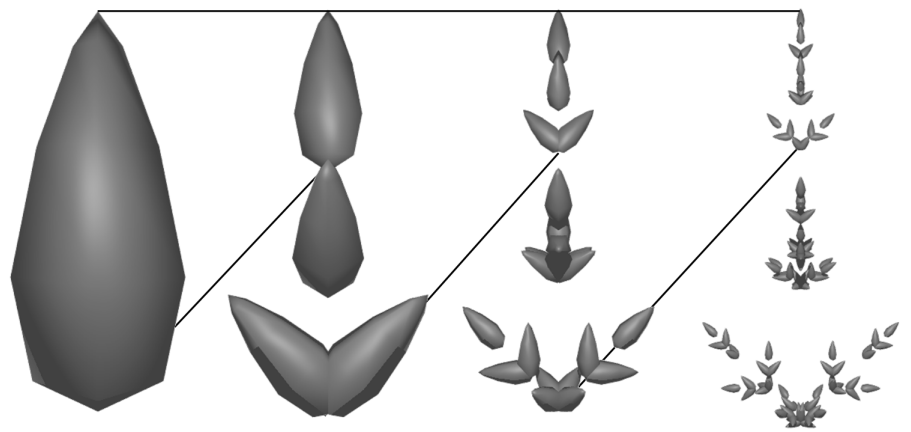

Fig. 2. Construction of an artificial crown. The initial object was a tapered ellipsoid and the IFS transformation was made of $n=5$ duplications of a contracted object by a factor $c=3$.

a stochastic 3D cantor dust was generated using a recursive algorithm derived from the method known as curdling and random trema generation [2 17]. Each iteration of the algorithm divides a given voxel into a set of subvoxels according to a specified subdivision factor. A fixed proportion of voxels eligible for the next iteration is chosen randomly from the subvoxels. At the end of the process, final voxels are considered to be leaves. The stochastic cantor dust is created by specifying a subdivision factor of 3 and $\frac{8}{27}$ as the proportion of chosen voxels for all 5 iteration levels. This object has the same theoretical dimension as the classical cantor dust.

\section{Estimation of the Fractal Dimension Using the BCM}

\subsection{The Box Counting Method}

The BCM has been extensively used to estimate fractal dimension of objects embedded in the plane. Its adaptation to 3D consists of building a sequence of $3 \mathrm{D}$ grids dividing space in homogeneous voxels of decreasing size $\delta$ and counting 

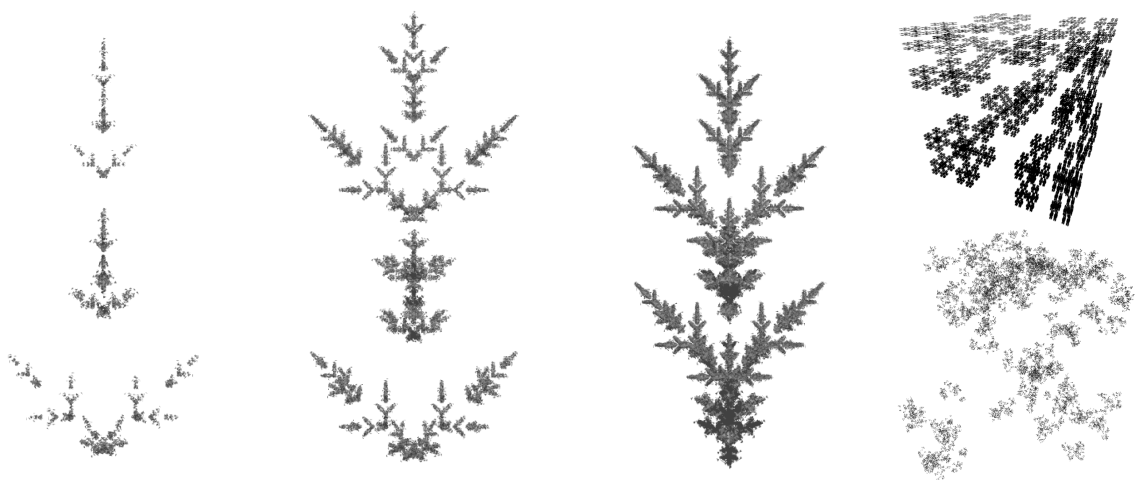

Fig. 3. From left to right, the three artificial canopies : AC1 $(n=5, c=3)$, AC2 $(n=7, c=3)$, AC3 $(n=9, c=3)$, on the top, the cantor dust and on the bottom a stochastic cantor dust

the number $N_{\delta}$ of grid voxels intercepted by the studied object. The estimator of the fractal dimension of the object is defined as

$$
D_{b}=\lim _{\delta \rightarrow 0} \frac{\log N_{\delta}}{\log \frac{1}{\delta}} .
$$

To implement this estimator, we approximated all the geometric objects by triangular meshes. The intersection of each triangle with the grid voxels can then be computed in time proportional to the number of triangles in the mesh [18. However, to decrease the overall complexity, we represent each triangle by a set of points [19. The number of points used is chosen such as the distance between two points is small compared to the minimal voxel size. The intersection algorithm is thus reduced to checking whether a voxel contains at least one point. The grid sequence is obtained by dividing the original bounding-box size, $\delta_{0}$, by a range of consecutive integers acting as subdivision factors. Thus the series of $\delta_{n}$ is a decreasing series formed by $\left\{\frac{\delta_{0}}{S_{i}}\right\}_{0 \leq i<n}$ where $S_{i}$ is the $i^{\text {th }}$ subdivision factor. Each sub-grid fits perfectly in the original bounding-box. It is important to note that several factors may influence the accuracy of this method, e.g. the choice of a proper range of scales and the orientation and alignement of the grid 20121]. In practice $D_{b}$ is estimated as the slope of the regression line between $\log N_{\delta}$ and $\log \frac{1}{\delta}$.

\subsection{Box Counting Method: Local Scale Variation Estimator}

As pointed out in [22], a major problem of the BCM estimator is that the numbers of intercepted voxels at each scale are correlated positively, and the correlation structure is completely ignored in the estimation procedure. This violates the assumption of data independency used in regression analysis. The consequence is an underestimation of confidence interval associated with the estimated fractal dimension. To eliminate the correlation, we introduce a new estimator, namely local 
scale variation estimator (LSV), based on the relative increase of intercepted voxels against the relative decrease in scale. This estimator can be derived from the BCM estimator as follows. Assuming the power law is verified for each scale $\delta$

$$
N_{\delta} \propto\left(\frac{1}{\delta}\right)^{D_{b}},
$$

the differential form of this equation leads to

$$
\begin{aligned}
d \log N_{\delta} & \propto d\left(D_{b} \log \left(\frac{1}{\delta}\right)\right), \\
\frac{d N_{\delta}}{N_{\delta}} & \propto-D_{b} \frac{d \delta}{\delta}
\end{aligned}
$$

which gives a variational interpretation of the fractal dimension. $D_{b}$ thus expresses the linear coefficient that corresponds to the ratio of new details due to a certain ratio of zoom in the structure. However, in this equation it is assumed that both $d N$ and $d \delta \simeq 0$, which is not usually the case for the scales used in BCM, except at very small scales. It is possible to generalize this variational principle to non-infinitely small quantities. Let $N_{\delta}$ be the number of intercepted voxels at scale $\delta$. We define $\Delta N_{\delta, \Delta \delta}$ as

$$
\Delta N_{\delta, \Delta \delta}=N_{\delta+\Delta \delta}-N_{\delta}
$$

The relative increase in the number of boxes is denoted $\widetilde{N}=\frac{\Delta N_{\delta, \Delta \delta}}{N_{\delta}}$. Similarly, we denote $\widetilde{\delta}=\frac{\Delta \delta}{\delta}$ the relative increase of zoom when passing from cell size $\delta$ to $\delta+\Delta \delta$. Thus, assuming Equation 3 is still satisfied, we have

$$
\widetilde{N} \propto \frac{(\delta+\Delta \delta)^{-D_{b}}-\delta^{-D_{b}}}{\delta^{-D_{b}}}=(1+\widetilde{\delta})^{-D_{b}}-1,
$$

which leads to a generalized form of Equation 4, where variations of $N_{\delta}$ and $\delta$ need not be infinitely small,

$$
\log (1+\widetilde{N}) \propto-D_{b} \log (1+\widetilde{\delta}) .
$$

$D_{b}$ can thus be estimated by performing a linear regression between $\log (1+\tilde{N})$ and $\log (1+\widetilde{\delta})$.

\section{Results}

\subsection{Number of Voxels as a Function of Scale}

In general, we may expect that the number of intercepted voxels is a monotonously increasing function of scale. However this is not always the case due to a quantization effect which results from discrepancy between discretization with the 3D grid and space occupation of the plant at some scales. Fig. 4 contains plots of the 
number of voxels intercepted at the different scales for each object. The local variation of the curves comes from the fact that the number of intercepted voxels at one scale depends of the adjustment of the grid. Some shiftings, up to a factor $\delta$ in each direction, and reorientations of the grid may lead to overestimating the number of voxels at one scale, causing local variation of the curve. Thus, the discrete quantization of the 3D shape of the object into voxels introduces some fuzziness in its representation, depending on scale. It can be seen in Fig. 4 that the quantization effect is far more pronounced with the artificial crowns and Cantor dusts than the digitized peach trees. This difference is attributed to the less deterministically distributed foliage of the digitized trees.
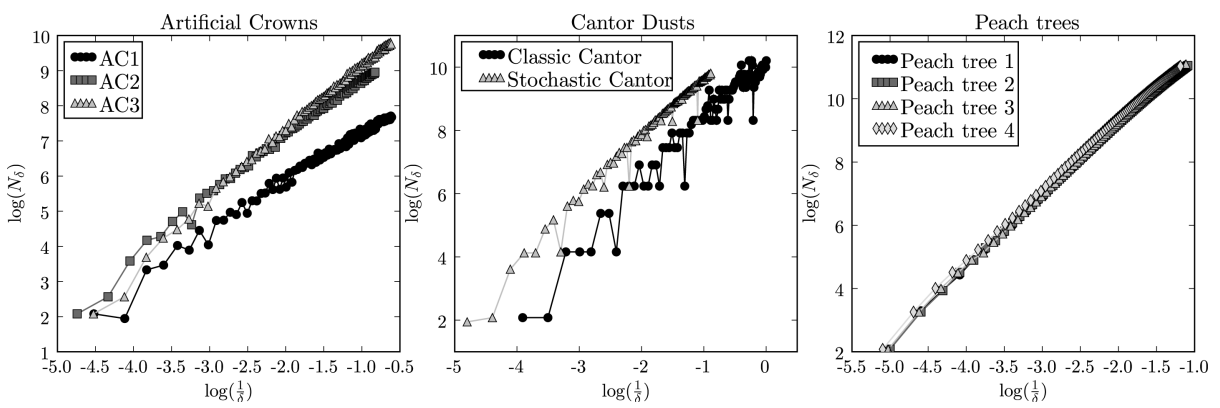

Fig. 4. The number of intercepted voxels as a function of the scale

\subsection{Estimating Fractal Dimension from the BCM}

Scale Range. When the grid voxel size is smaller than the leaf size, the evaluation of the dimension is modified by the dimension of the leaves surfaces. To avoid this effect, a minimum voxel size, $\delta_{\text {min }}$, is determined such as $\delta_{\text {min }} \geq \sqrt{\mathcal{A}_{l}}$, where $\mathcal{A}_{l}$ is the mean leaf area. Since every voxel size $\delta_{i}$ is obtained from the bounding box size $\delta_{0}$ as $\delta_{i}=\frac{\delta_{0}}{S_{i}}$, the minimum size must be $\delta_{\min }=\frac{\delta_{0}}{S_{\max }}$. Let $\mathcal{V}_{b b}$ be the bounding box volume. An uni-dimensional proportionality factor is defined by

$$
S_{\text {max }}=\frac{\sqrt[3]{\mathcal{V}_{b b}}}{\sqrt{\mathcal{A}_{l}}} .
$$

Setting $S_{\max }$ as the upper bound for the subdivision factors $\left\{S_{i}\right\}_{0 \leq i<n}$ guaranties that no voxel size will be smaller than a leaf size.

Grid Shifting. When the voxel size is close to the leaf size, the local adjustments of the grid may cause significant variations in the number of intercepted voxels, as discussed above. Practically, to limit the effect of this local variation due to grid shifting, a factor $\frac{S_{\max }}{3}$ instead of $S_{\max }$ as the contraction limit was considered. This factor can be explained as follows. Let us consider a grid with voxels equal in size to the mean leaf size. Optimally a leaf will be included into a single voxel. All 
the possible shifting configurations of the grid may cause the leaf also be included in any of the twenty-six neighboring voxels. Considering voxels of bigger sizes with a factor 3 can be seen as including the twenty-seven possible small voxels into the same large one and so limits the errors found in finer grids. Of course, the optimal grid for one leaf will not be the optimal grid for all leaves; therefore, artifact effects of grid adjustment may persist. We experimentally observed that this persistence is limited (see Fig. 5).

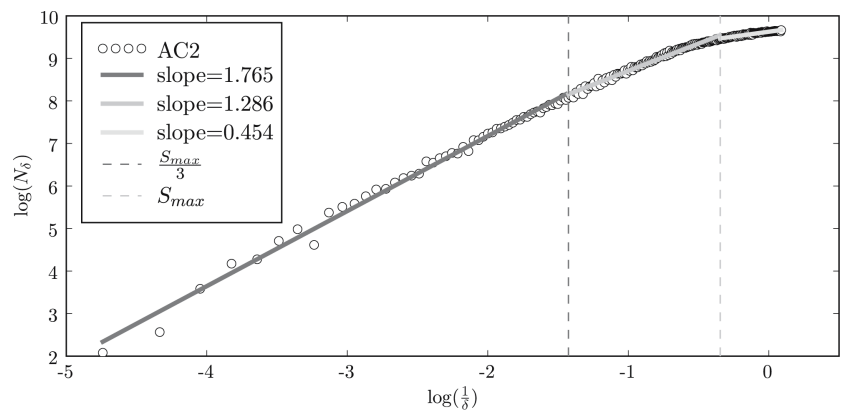

Fig. 5. Evolution of AC2 slope during BCM evaluation. The number of voxels intercepted at various scales for AC2 with the slopes highlighted. In the range $\left[0, \frac{S_{\max }}{3}\right]$, the slope is primarily influenced by the structure of AC2 and the fractal dimension $D_{b}=$ 1.765. In the range $\left[\frac{S_{\max }}{3}, S_{\max }\right]$, the slope is also partially influenced by the fractal dimension of individual leaves and is sensible to local variation due to grid adjustment. When this range is taken into account for the fractal dimension evaluation, $D_{b}$ drops from 1.765 to 1.584 . Finally for grids with voxel sizes smaller than $S_{\max }$, the slope is directly related to individual leaf fractal dimension ( 0 in our representation since we use points). With a naive range of evaluation including all points, the fractal dimension drops to 1.172 .

Orientation of the Grid. Optimal voxel coverage of the plant depends on the orientation of the grid relative to the plant. For this, we made a sensitivity analysis to evaluate how the estimated fractal dimension is affected by changes in the grid's orientation. A set of random grid orientations were selected and fractal dimension was estimated for each orientation. Table 1 gives the mean and variance of the estimated fractal dimension across orientations for all the considered plants. We can observe a low variability in the absolute values of the results: the standard deviations are inferior to one per cent of the mean values. From this, we conclude that the orientation of the grid has only limited effect on the BCM evaluation method.

Error Characterization. To characterize the error made during the estimation, a comparison with theoretical fractal dimension can be used. In the case of plants corresponding to IFS attractors, the theoretical fractal dimension, $D$, is known. But there is no such dimension for real plants; however, it has been shown that, 
when plant's topology is known, a faithful estimate of the plant fractal dimension can be obtained using the two-surface method [23]. This value will be used as reference value for the peach trees.

A classical Student's t-test on the computed $D_{b}$ distributions shows that a significant bias in the BCM estimation exists. However, results reported in Table 1 (cols 3-6) show that this bias is less than $3.1 \%$ of the theoretical value for the studied canopies.

Table 1. Fractal dimension results for studied canopies and their properties. $D_{a}$ is the reference (theoretical) value of the fractal dimension. For $D_{b}$ estimation, $\overline{D_{b}}$ gives the mean estimated value and $\sigma$ the standard deviation over all considered rotations. The minimum standard error $r^{2}$ over all rotations is shown. All results are obtained with $\frac{S_{\max }}{3}$ as the upper limit.

\begin{tabular}{cc|ccc|c|ccc|ccc}
\hline & & \multicolumn{3}{|c|}{ BCM $D_{b}$} & Relative & \multicolumn{3}{|c|}{ LSV $D_{b}$} & & & \\
Canopy & $D_{a}$ & $\overline{D_{b}}$ & $\sigma$ & $r^{2}$ & Bias & $D_{b}$ & $\sigma$ & $r^{2}$ & $\sqrt[3]{\mathcal{V}_{b b}}$ & $\sqrt{\mathcal{A}_{l}}$ & $S_{\text {max }}$ \\
\hline AC1 & 1.47 & 1.4889 & 0.0056 & 0.97 & 0.0128 & 1.8761 & 0.0457 & 0.33 & 1.83 & 0.0143 & 128 \\
AC2 & 1.77 & 1.7305 & 0.0053 & 0.99 & 0.0223 & 1.9409 & 0.06 & 0.58 & 2.29 & 0.0143 & 160 \\
AC3 & 2 & 1.97 & 0.0074 & 0.99 & 0.015 & 2.0705 & 0.0534 & 0.74 & 1.85 & 0.0143 & 129 \\
\hline Cantor & 1.89 & 1.8835 & 0.0174 & 0.94 & 0.0034 & 2.2286 & 0.0852 & 0.09 & 0.99 & 0.0041 & 243 \\
Stoc. Cantor 1.89 & 1.8896 & 0.0105 & 0.97 & 0.0002 & 2.1218 & 0.0933 & 0.17 & 2.43 & 0.01 & 243 \\
\hline Peach 1 & 2.33 & 2.3221 & 0.0043 & 0.99 & 0.0033 & 2.2832 & 0.0115 & 0.97 & 2.97 & 0.439 & 67 \\
Peach 2 & 2.36 & 2.3516 & 0.0056 & 0.99 & 0.0035 & 2.3416 & 0.0117 & 0.97 & 2.97 & 0.459 & 64 \\
Peach 3 & 2.38 & 2.307 & 0.0064 & 0.99 & 0.0306 & 2.3022 & 0.0195 & 0.97 & 3.04 & 0.0463 & 65 \\
Peach 4 & 2.33 & 2.3218 & 0.0076 & 0.99 & 0.0035 & 2.3147 & 0.0175 & 0.98 & 2.61 & 0.0449 & 72 \\
\hline
\end{tabular}

\subsection{Estimating Fractal Dimension from the LSV Method}

We use the LSV estimator of the box counting method, presented on section 3.2. on the theoretical and digitized plants. The $\widetilde{\delta}$ values were defined using couple of successive scales

$$
\widetilde{\delta}=\frac{\delta_{i+1}-\delta_{i}}{\delta_{i}}=\frac{1}{\delta_{i}}
$$

and $\widetilde{N}$ values from the corresponding $N$ values. Since it is based on a local estimation, it is sensible to the local variation of the number of box as a function of scales introduced by the quantization effect. The local variations in this estimation are reflected in the variance and standard error of the computed fractal dimensions, giving a better estimation of the reliability of the results compared to the classical box counting method.

Experimentally, we observe that results on theoretical plants are very sensitive to quantization effect as shown by dispersion of the data in the Fig. 6 and the minimum standard error in Table 1 (cols 7-9). The minimum $r^{2}$ for the estimated dimensions on these objects are between 0.09 to 0.74 . This effect is much less important on real plants; the minimum $r^{2}$ values are between 0.97 and 0.98 . In this case, the results seems more relevant. The difference with theoretical values is small (less than $3.2 \%$ ). 

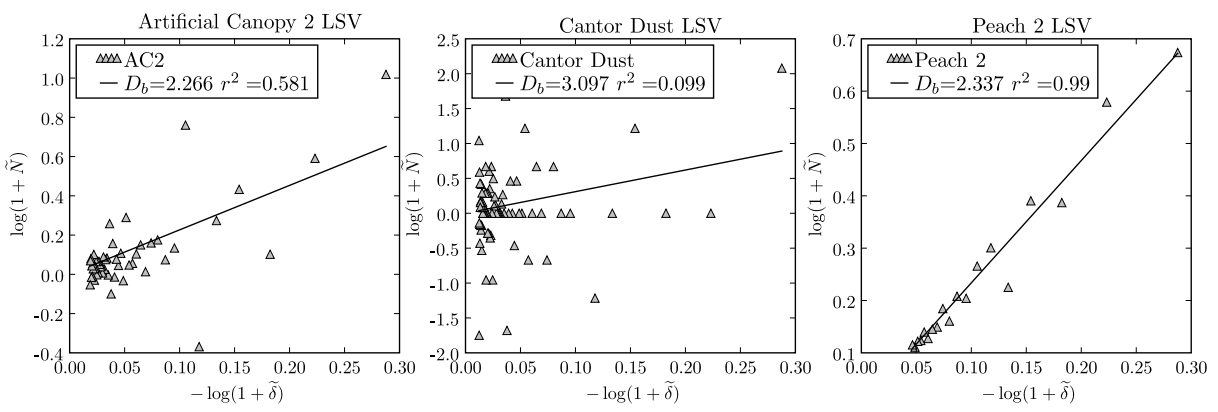

Fig. 6. Estimated fractal dimension with the LSV method for AC2, Cantor Dust and Peach 2. This new estimator is very sensitive to quantization effect leading to a dispersion of the measurements in AC2 and Cantor Dust. On the contrary the method gives an estimation of $D$ close to that obtained with the two-surface method (i.e $D=2.36$ ) for Peach 2 tree.

\section{Conclusion}

In this paper the accuracy of the BCM for evaluating the fractal dimension of 3D crowns was studied. Several factors that may influence this accuracy were examined and practical solutions proposed. In particular a proper voxel size limit is determined dependent on leaf sizes and the BCM bias was quantified. The problem of data dependency used during the regression analysis was discussed and a new estimator, LSV, that does not violate the independence assumption is described. The LSV estimator appears to be an interesting indicator to determine whether the quantization effect disturb the fractal dimension estimation. Eventually it has to be improved to support more robust evaluations.

\section{References}

1. Godin, C.: Representing and encoding plant architecture: A review. Annals of Forest Science 57 (2000) 413-438

2. Mandelbrot, B.B.: The fractal geometry of nature. Freeman (1983)

3. Smith, A.R.: Plants, fractals, and formal languages. In: Siggraph'84, Computer Graphics Proceedings. Volume 18., ACM Press (1984) 1-10

4. Barnsley, M.: Fractals Everywhere. Academic Press, Boston (1988)

5. Prusinkiewicz, P., Hanan, J.: Lindenmayer systems, fractals, and plants. Lecture Notes in Biomathematics 75 (1989)

6. Chen, S., Ceulemans, R., Impens, I.: A fractal-based Populus canopy structure model for the calculation of light interception. Forest Ecology and Management 69(1-3) (1994) 97-110

7. Prusinkiewicz, P., Mundermann, L., Karwowski, R., Lane, B.: The use of positional information in the modeling of plants. In: Siggraph'01, Computer Graphics Proceedings, New York, NY, USA, ACM Press (2001) 289-300

8. Fitter, A.H.: An architectural approach to the comparative ecology of plant root systems. New Phytologist 106(1) (1987) 61-77 
9. Eshel, A.: On the fractal dimensions of a root system. Plant, Cell \& Environment 21(2) (1998) $247+$

10. Oppelt, A.L., Kurth, W., Dzierzon, H., Jentschke, G., Godbold, D.L.: Structure and fractal dimensions of root systems of four co-occurring fruit tree species from Botswana. Annals of Forest Science 57 (2000) 463-475

11. Morse, D.R., Lawton, J.H., Dodson, M.M., Williamson, M.H.: Fractal dimension of vegetation and the distribution of arthropod body lengths. Nature 314(6013) (1985) 731-733

12. Critten, D.L.: Fractal dimension relationships and values associated with certain plant canopies. Journal of Agricultural Engineering Research 67(1) (1997) 61-72

13. Falconer, K.: Fractal geometry : mathematical foundation and applications. John Wiley and Sons (1990)

14. Boudon, F., Pradal, C., Nouguier, C., Godin, C.: Geom module manual: I user guide. Technical Report 3, CIRAD (2001)

15. Sonohat, G., Sinoquet, H., Kulandaivelu, V., Combes, D., Lescourret, F.: Threedimensional reconstruction of partially $3 \mathrm{~d}$-digitized peach tree canopies. Tree Physiol 26(3) (2006) 337-351

16. Falconer, K.: Techniques in fractal geometry. John Wiley and Sons (1997)

17. Plotnick, R.E., Gardner, R.H., O'Neill, R.V.: Lacunarity indices as measures of landscape texture. Landscape Ecology 8 (1993) 201-211

18. Andres, E., Nehlig, P., Franon, J.: Supercover of straight lines, planes and triangles. In: Proceedings of DGCI '97, London, UK, Springer-Verlag (1997) 243-254

19. Pfister, H., Zwicker, W., Baar, J.v., Gross, M.: Surfels: surface elements as rendering primitives. In: Siggraph'00, Computer Graphics Proceedings, Los angeles, ACM Press (2000) 335-342

20. Foroutan-Pour, K., Dutilleul, P., Smith, D.L.: Advances in the implementation of the box-counting method of fractal dimension estimation. Applied Mathematics and Computation 105(2) (1999) 195-210

21. Halley, J.M., Hartley, S., Kallimanis, A.S., Kunin, W.E., Lennon, J.J., Sgardelis, S.P.: Uses and abuses of fractal methodology in ecology. Ecology Letters 7 (2004) $254-271$

22. Reeve, R.: A warning about standard errors when estimating the fractal dimension. Comput. Geosci. 18(1) (1992) 89-91

23. Boudon, F., Godin, C., Pradal, P., Puech, O., Sinoquet, H.: Estimating the fractal dimension of plants using the two-surface method. an analysis based on 3d-digitized tree foliage. Fractals 14(3) (2006) 